\title{
Isotopically Resolved Photoelectron Imaging Unravels Complex Atomic Autoionization Dynamics by Two-Color Resonant Ionization
}

\author{
P. O'Keeffe, ${ }^{1}$ E. V. Gryzlova, ${ }^{2}$ D. Cubaynes, ${ }^{3}$ G. A. Garcia, ${ }^{4}$ L. Nahon, ${ }^{4}$ A. N. Grum-Grzhimailo, ${ }^{2}$ and M. Meyer ${ }^{5}$ \\ ${ }^{1}$ CNR Istituto di Metodologie Inorganiche e dei Plasmi, CP10, I-00016 Monterotondo Scalo, Italy \\ ${ }^{2}$ Skobeltsyn Institute of Nuclear Physics, Lomonosov Moscow State University, Moscow 119991, Russia \\ ${ }^{3}$ ISMO, CNRS/Université Paris-Sud, Bâtiment 350, F-91405 Orsay Cedex, France \\ ${ }^{4}$ Synchrotron SOLEIL, L'Orme des Merisiers, St. Aubin, B.P. 48, 91192 Gif sur Yvette, France \\ ${ }^{5}$ European XFEL GmbH, Albert-Einstein-Ring 19, D-22761 Hamburg, Germany
}

(Received 26 September 2013; published 9 December 2013)

\begin{abstract}
Angle-resolved electron spectroscopy in coincidence with high-resolution mass spectroscopy has been applied to study two-color resonant photoionization in atomic xenon. Separation of different isotopes enabled us to extract results for the electronic dynamics free from depolarization effects, which are generally introduced by the coupling of the electronic and nuclear angular momenta. The concerted experimental and theoretical analysis of the photoelectron angular distributions in the region of an autoionizing resonance emphasizes the strong sensitivity of the observed structures to the fine details of the treatment of the underlying dynamics.
\end{abstract}

DOI: 10.1103/PhysRevLett.111.243002

PACS numbers: $32.80 . \mathrm{Fb}, 32.80 . \mathrm{Rm}, 32.80 . \mathrm{Zb}$

Resonant photoionization and the underlying electron dynamics are the subject of numerous experimental and theoretical studies. Since Fano demonstrated [1] that the asymmetric shape of the resonances results from quantum interferences between resonant (discrete) and direct (continuum) photoionization processes, the main effort has focused on the identification of the relevant ionization channels and on the best possible control of the electronic states involved. Advanced experimental techniques based, for example, on ultrahigh spectral resolution of the ionizing radiation [2,3], on two-photon excitation schemes (e.g., $[4,5])$, on excitation from metastable atoms (e.g., [6]), on the analysis of the angular distribution or spin of the outgoing electrons (e.g., [7,8]), and, very recently, laser control of absorption line shapes [9] have been applied to obtain information on the complex electron dynamics. However, detailed comparison with theoretical approaches is often hindered by the fact that the experimental results represent an average over isotopes with different nuclear spins. In the case of atoms with nonzero nuclear spin the carefully prepared polarized intermediate state can undergo a depolarization due to coupling of its electronic angular momentum with the nuclear spin. From a quantum mechanical view, the coherent excitation of the hyperfine levels of the intermediate state leads to a mixing of states with different magnetic quantum numbers $m$ and to a temporal evolution of the resulting nonstationary state (quantum beats). The effect of this process on the photoelectron angular distributions (PADs) was first observed by Berry and co-workers in the two-photon ionization of sodium and lithium [10,11] and subsequently studied in other systems (e.g., [12-15]). These studies showed that detailed knowledge of the hyperfine structure and of the temporal profiles of both the excitation and the ionization photon sources is required to fully understand the depolarization due to hyperfine interactions. Also, in the case of two-photon ionization of $\mathrm{Ba}$ via an intermediate state, it was shown that transitions to certain final-state resonance series are only possible due to the hyperfine depolarization of the intermediate state (e.g., $[16,17])$. Generally, it is recognized that even though the coupling of the electron angular momentum with the nuclear spin is weak, it has important consequences for many physical applications (e.g., [18]), and especially for the photoionization process.

In this Letter, we show that such a coupling in the case of autoionizing doubly resonant excited states of Xe, may have a dramatic impact on the resulting measured PADs. Therefore, it is only by filtering the zero nuclear spin contribution to the circular dichroism (CD) and PAD data that the pure electronic aspects of photoionization dynamics can be unraveled by comparison with theory. In order to separate the effects of the nuclear spin in the present study, we have measured the PADs in coincidence with the isotope selected ions. Using a two-photon double-resonant excitation scheme a polarized ensemble of $\mathrm{Xe}^{*} 5 d\left[\frac{3}{2}\right]_{1}$ atoms was prepared by photoexcitation from the ground state and ionized in the region of the autoionizing $\mathrm{Xe}^{*} 4 f^{\prime}\left[\frac{5}{2}\right]_{2}$ resonance (see Fig. 1) [19]. The complex dynamics of this resonant process and the high sensitivity of its description to the theoretical model was previously outlined $[20,21]$.

The experiments were performed at the vacuum ultraviolet (VUV) variable polarization beam line, DESIRS [22], of the French synchrotron source, SOLEIL, whose scientific case includes the study of photoionization dynamics and $\mathrm{CD}$, together with the permanently installed molecular beam chamber SAPHIRS. A supersonic beam of xenon atoms was formed by expansion through a $50 \mu \mathrm{m}$ 


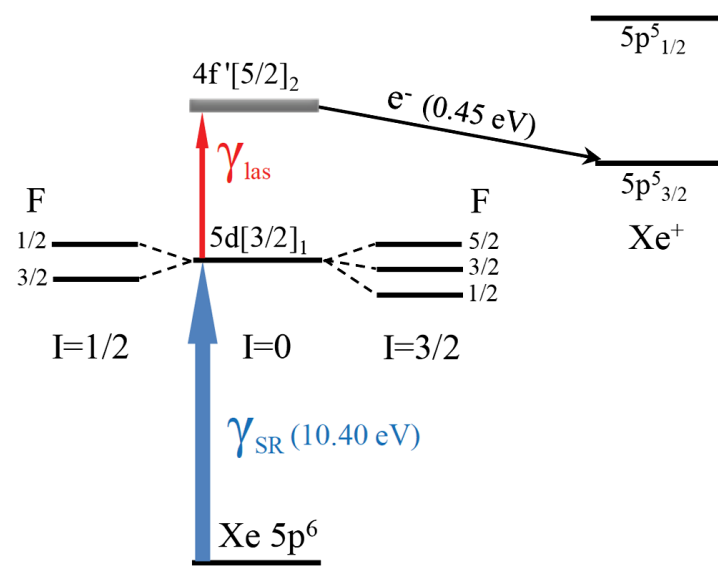

FIG. 1 (color online). Schematic diagram of the process described in this article, showing the hyperfine structure (HFS) of the intermediate $\mathrm{Xe}^{*} 5 p_{3 / 2}^{5} 5 d\left[\frac{3}{2}\right]_{1}$ state for atoms with nuclear spin $I=0, \quad I=\frac{1}{2}$, and $I=\frac{3}{2}$. The HFS levels of the $\mathrm{Xe}^{*} 5 p_{1 / 2}^{5} 4 f^{\prime}\left[\frac{5}{2}\right]_{2}$ state overlap due to its broad autoionization width, while the HFS of the $\mathrm{Xe}^{+} 5 p_{j}^{5}$ is not relevant for the present discussion.

nozzle and its central part selected by a skimmer, before crossing the counterpropagating synchrotron radiation (SR) and laser photon beams at right angles at the center of the ionization chamber. After excitation by the VUV photons to the intermediate $5 d\left[\frac{3}{2}\right]_{1}$ resonance, the excited atoms were subsequently excited to the autoionizing $4 f^{\prime}\left[\frac{5}{2}\right]_{2}$ state by a conventional continuous linear dye laser pumped by the $532 \mathrm{~nm}$ light of a frequency-doubled solid state laser (5W VERDI). Using a Rhodamine $6 \mathrm{G}$ dye an average laser power of $800 \mathrm{~mW}$ and a spectral width of about $1.3 \mathrm{~cm}^{-1}$ was obtained in the maximum of the emission curve of the dye $(595 \mathrm{~nm})$. The resulting ions and electrons were accelerated in opposite directions perpendicular to the molecular and photon beams inside the Delicious II angle-resolving photoelectron-photoion coincidence imaging spectrometer [23] which combines a velocity mapping imaging (VMI) spectrometer and a linear Wiley McLaren-type ion time of flight spectrometer. The spectrometer was operated in the coincidence mode so that each electron could be binned according to the flight time (and consequently mass) of the ion formed in the same event. Figure 2 presents a typical time of flight spectrum showing that the various natural isotopes of Xe can be easily separated by the apparatus. In this way, the velocity mapped photoelectron image for each isotope can be built up. These images were then inverted using the PBASEX software [24] in order to extract the parameters describing the electron angular distributions resulting from ionization of the $I=0\left({ }^{132,134,136} \mathrm{Xe}\right), I=$ $\frac{1}{2}\left({ }^{129} \mathrm{Xe}\right)$, and $I=\frac{3}{2}\left({ }^{131} \mathrm{Xe}\right)$ atoms.

In the present study, we limit ourselves to the cases of circularly polarized photon beams and parallel linearly polarized photon beams. The $Z$ axis is directed along the SR beam for the processes with circularly polarized photons

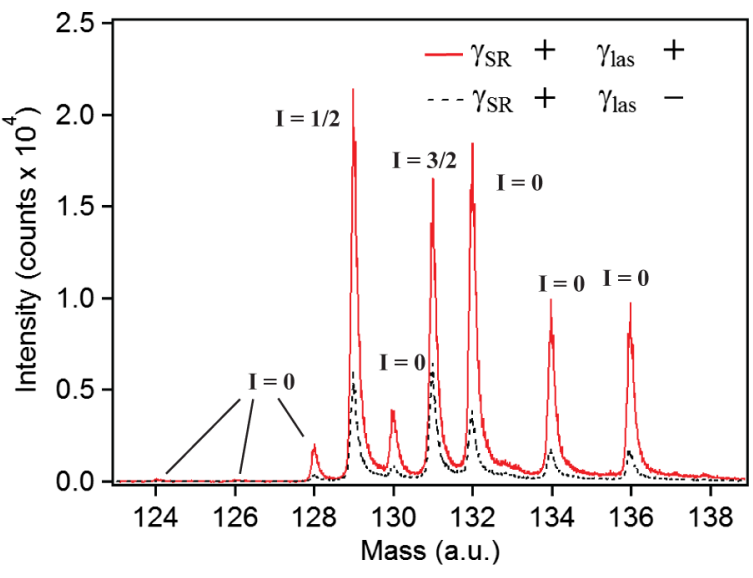

FIG. 2 (color online). The mass spectra of the $\mathrm{Xe}^{+}$ions are shown for two-photon ionization via the resonant $\mathrm{Xe} 5 p^{6}+$ $\gamma_{\mathrm{SR}} \rightarrow 5 d\left[\frac{3}{2}\right]_{1}+\gamma_{\text {las }} \rightarrow 4 f^{\prime}\left[\frac{5}{2}\right]_{2}$ pathway with right $(+)$ circularly polarized SR and both right $(+)$ and left $(-)$ circularly polarized visible laser light.

and along the direction of the polarization for the process with the linearly polarized photons. Then within the dipole approximation the PADs can be cast into the form

$$
\left(\frac{d \sigma}{d \Omega}\right)_{\nu \nu^{\prime}}=\frac{\sigma^{\nu \nu^{\prime}}}{4 \pi}\left(1+\beta_{2}^{\nu \nu^{\prime}} P_{2}(\cos \theta)+\beta_{4}^{\nu \nu^{\prime}} P_{4}(\cos \theta)\right),
$$

where $\sigma^{\nu \nu^{\prime}}$ is the angle-integrated photoionization cross section, $\beta_{k}^{\nu \nu^{\prime}}(k=2,4)$ the asymmetry parameters, and $P_{k}(x)$ are the Legendre polynomials. The superscript $\nu \nu^{\prime}$ indicates the polarization state of the photons: ++ and +for parallel and antiparallel spins of the circularly polarized photons, respectively (the first sign corresponds to the SR, the second to the laser, and "+ " is assigned to the direction of the $Z$ axis), and $\nu \nu^{\prime}=$ lin for linearly polarized photons. The $C D$ is then defined as

$$
\mathrm{CD}=\left(\sigma^{++}-\sigma^{+-}\right) /\left(\sigma^{++}+\sigma^{+-}\right) .
$$

To treat theoretically the PAD and the $\mathrm{CD}$ in the region of an isolated autoionizing resonance, we apply the formalism of [25]. Using this methodology, the quantities in Eqs. (1) and (2) are expressed in terms of the reduced matrix elements of the dipole operator connecting the $5 d\left[\frac{3}{2}\right]_{1}$ state with the autoionizing state $4 f^{\prime}\left[\frac{5}{2}\right]_{2}$ and the adjacent continuum states $5 p_{3 / 2}^{5} \epsilon \ell$, where $\epsilon$ and $\ell$ are photoelectron energy and orbital momentum, respectively, the matrix elements describing the decay of the $4 f^{\prime}\left[\frac{5}{2}\right]_{2}$ state into the continuum due to the Coulomb interaction, and the orientation $\left(\mathcal{A}_{10}^{\nu}\right)$ and alignment $\left(\mathcal{A}_{20}^{\nu}\right)$ of the angular momentum $J$ of the electronic shell in the $5 d\left[\frac{3}{2}\right]_{1}$ state excited by linearly $(\nu=$ lin $)$ or right circularly $(\nu=+)$ polarized SR [26]. It is the atomic orientation and the alignment parameters which are decreased by the depolarization in a difficult to control manner. Figure 3 shows experimentally the effect of this depolarization on 

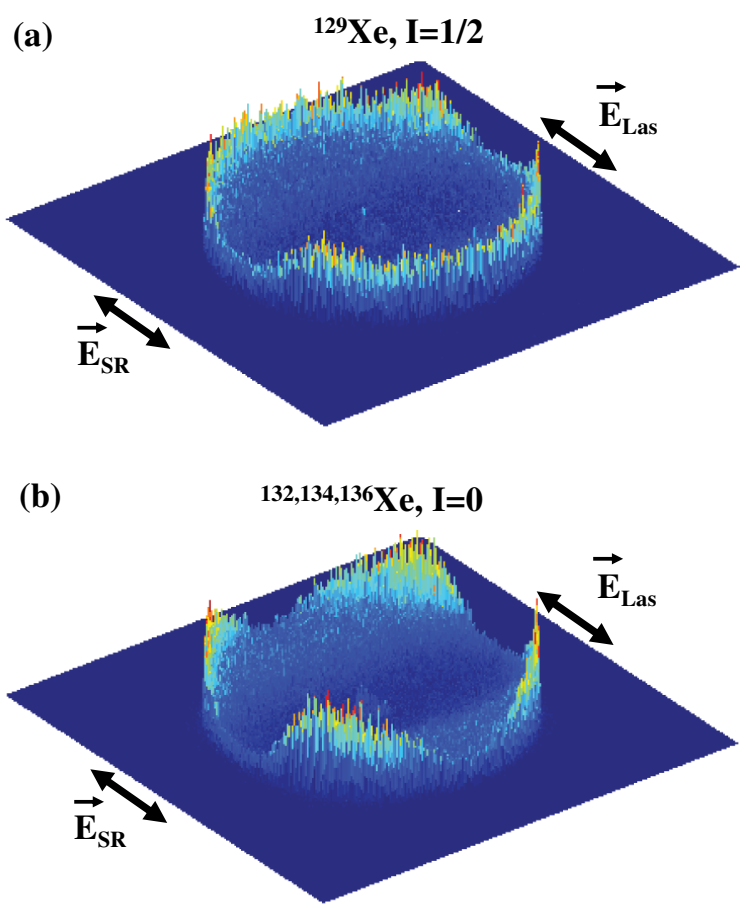

FIG. 3 (color online). Panels (a) and (b) contain the raw VMI images for parallel linear polarizations of both SR and optical laser light of the photoelectrons taken in coincidence with the (a) ${ }^{129} \mathrm{Xe}^{+}$and (b) ${ }^{132,134,136} \mathrm{Xe}^{+}$ions. The images are shown as $3 \mathrm{D}$ representations in order to visualize clearly the differences in the anisotropy of the angular distributions.

the PADs by comparing the VMI photoelectron images recorded with linear polarizations in coincidence with the $\mathrm{Xe}^{+}\left(I=\frac{1}{2}\right)$ and $\mathrm{Xe}^{+}(I=0)$ ions with the laser tuned to the maximum of the $4 f^{\prime}\left[\frac{5}{2}\right]_{2}$ resonance. It is quite clear by simple visual inspection that the PAD corresponding to $I=0$ ions is much more structured than that of the $I=\frac{1}{2}$ image. To our knowledge this is the first time that the PADs due to ionization of zero and nonzero nuclear spin atoms have been separated experimentally.

The numerical data for the asymmetry parameters were obtained within an intermediate-coupling multiconfigurational Hartree-Fock approximation (MCHF) [27]. Within the MCHF approach, $L S$-coupled wave functions are used as the basis in the multiconfiguration expansion for the wave functions of the initial photoexcited and the autoionizing states of Xe with the total angular momentum $J$ and parity $\pi$ in terms of configuration state functions (CSFs): $|p, \pi J\rangle=\sum_{i=1} C_{i}^{p}\left|\alpha_{i}, \pi L_{i} S_{i}\right\rangle$. Here the label $p$ numerates the states for distinction, $\alpha_{i}$ denotes the occupation of the different subshells (configuration), and their angular couplings $L_{i}$ and $S_{i}$ stand for the orbital angular momentum and the spin of the CSF, respectively, and $C_{i}^{p}$ are the mixing coefficients for the state $p$. The electron orbitals were generated with the frozen $\mathrm{Xe}^{+} 5 p^{5}$ core. We accounted for the $5 p^{5}(6-9) s,(5-7) d, 6 p, 4 f, 5 f$ configurations. The set of configurations with the appropriate parity $\pi$ was used to find the coefficients $C_{i}^{p}$ by the diagonalization of the Breit-Pauli atomic Hamiltonian. The wave functions $\epsilon \ell(\ell=1,3)$ of the electron in the continuum were calculated within the frozen $5 p^{5}$ core term-dependent approximation with orthogonalization to all bound orbitals of the same symmetry.

Without taking into account any depolarization of the electronic-shell angular momentum of the $\mathrm{Xe}^{*} 5 d\left[\frac{3}{2}\right]_{1}$ state, the calculated value for the $\mathrm{CD}$ is 0.68 , in excellent agreement with the experimental value of $0.67 \pm 0.02$ for the isotopes with zero nuclear spin, extracted from the experimental data shown in Fig. 2. This result for the $I=0$ isotope points also to negligible depolarization of the $\mathrm{Xe}^{*}$ atoms in the reaction volume due to reasons other than hyperfine interaction. However, the measured reduced $\mathrm{CD}=0.54 \pm 0.04$ and $0.43 \pm 0.03$ for the isotopes ${ }^{129} \mathrm{Xe}\left(I=\frac{1}{2}\right)$ and ${ }^{131} \mathrm{Xe}\left(I=\frac{3}{2}\right)$, respectively, clearly show that for isotopes with nonzero nuclear spin depolarization of the electronic shell due to the hyperfine interactions is rather large, and accounts for the corresponding much less structured PAD of Fig. 3(a).

Figure 4 shows a comparison between the depolarization-free measurements of the asymmetry

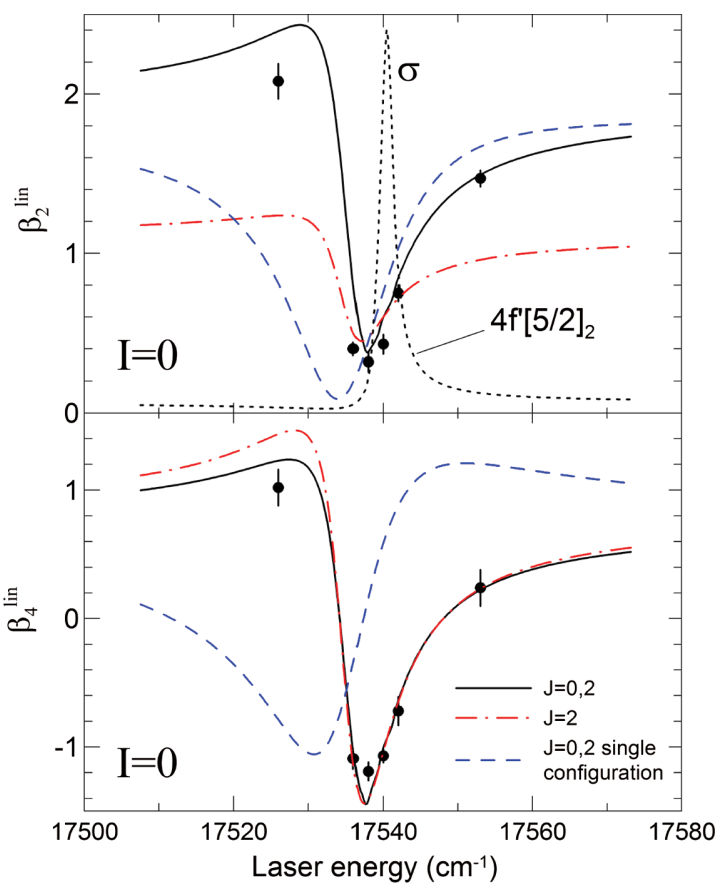

FIG. 4 (color online). The panels show the $\beta_{2}^{\text {lin }}$ (top) and $\beta_{4}^{\text {lin }}$ (bottom) parameters for Xe ions with nuclear spin $I=0$ at different laser wavelengths across the $5 d\left[\frac{3}{2}\right]_{1}+\gamma_{\text {las }} \rightarrow 4 f^{\prime}\left[\frac{5}{2}\right]_{2}$ resonance. The experimental data (black circles) are extracted from the VMI images recorded in coincidence with $I=0$ ions $\left({ }^{132,134,136} \mathrm{Xe}^{+}\right)$. The theoretical results are obtained using different approximations: MCHF (solid); MCHF only with $J=2$ dominating channels (chain); single configuration (dashed). The profile of the $4 f^{\prime}\left[\frac{5}{2}\right]_{2}$ resonance in the angle-integrated cross section $\sigma$ is shown for comparison in the top panel (dotted line). 
parameters across the $4 f^{\prime}\left[\frac{5}{2}\right]_{2}$ resonance and the theoretical predictions. The profiles for $\beta_{k}^{\text {lin }}$, with $k=2$ and 4 , are equally broadened and slightly shifted with respect to the profile of the resonance in the angle-integrated cross section in accordance with the scaling theorem described in [28].

The autoionizing state $4 f^{\prime}\left[\frac{5}{2}\right]_{2}$ dominantly decays with the emission of $\epsilon f$ electrons, despite competition between two opposite trends. On the one hand, the overlap between radial components of the $4 f$ and $\epsilon f$ electron wave functions, governing the decay amplitude, is much larger than that between the $4 f$ and $\epsilon p$ functions. On the other hand, the decay $5 p_{1 / 2}^{5} 4 f\left[\frac{5}{2}\right]_{2} \rightarrow 5 p_{3 / 2}^{5} \epsilon f$ (due to the Coulomb interaction) is suppressed by a selection rule. The latter prohibits $j K-L S J$ transitions with equal orbital moments of the outer electron for a fixed $L S$ term of the ionic core $\mathrm{Xe}^{+} 5 p^{52} p$ in our case). Therefore, the decay into the dominating $\epsilon f$ channel proceeds in our model due to a tiny violation of the pure $j K$-coupling scheme for the $4 f^{\prime}\left[\frac{5}{2}\right]_{2}$ state (the coefficients $C_{i}^{p}$ differ from those for the $j K$ coupling by less than 0.006 , which is below $1 \%$ ) and a very small admixture $(<0.05 \%)$ of the $5 p^{5} n p$ states. The delicate balance between different terms of the decay amplitude is the reason for its very high sensitivity to the model. Similarly, the amplitude of the photoexcitation $5 d\left[\frac{3}{2}\right]_{1} \rightarrow 4 f^{\prime}\left[\frac{5}{2}\right]_{2}$ varies crucially with the inclusion of higher configurations. Our single-configuration results do not reproduce the measured asymmetry parameters (see Fig. 4), whereas in the case of the angle-integrated cross section this model provides results close to those measured experimentally in [21]. Generally, ionization from the Xe $\mathrm{Xe} 5 d\left[\frac{3}{2}\right]_{1}$ state proceeds into the channels $5 p_{3 / 2}^{5} \epsilon p_{1 / 2}(J=$ $1,2), 5 p_{3 / 2}^{5} \epsilon p_{3 / 2}(J=0,1,2), 5 p_{3 / 2}^{5} \epsilon f_{5 / 2}(J=1,2)$, and $5 p_{3 / 2}^{5} \epsilon f_{7 / 2}(J=2)$. Because of the parallel polarizations of the two photons and absence of depolarization of the $5 d\left[\frac{3}{2}\right]_{1}$ state for $I=0$ isotopes, the ionization channels with the total angular momentum $J=1$ do not contribute. Therefore, only five ionization channels have to be considered: four "resonant" channels with $J=2$, which interact with the autoionizing state $4 f^{\prime}\left[\frac{5}{2}\right]_{2}$, and one "weak" nonresonant channel with $J=0$. Interference of the channels with different total angular momentum $J$, although it does not contribute to the angle integrated cross section, influences the PAD (e.g., [25]). The theoretical predictions for the $\beta_{2}^{\text {lin }}$ parameter (Fig. 4) are significantly improved when switching on the weak ionization channel with $J=0$ due to its interference with the $J=2$ channels. In contrast, the above interference is lacking in $\beta_{4}^{\text {lin }}$ due to a triangle rule $\left|J-J^{\prime}\right| \leq k \leq J+J^{\prime}$, and the $J=0$ channel alters only slightly via the small corresponding partial photoionization cross section (Fig. 4). In general, good agreement between experiment and theory is achieved in our MCHF calculations for the PADs, where the simpler models fail.

In conclusion, we have studied the photoionization dynamics of the doubly resonant $5 p^{6} \rightarrow 5 d\left[\frac{3}{2}\right]_{1} \rightarrow 4 f^{\prime}\left[\frac{5}{2}\right]_{2}$ excitation in atomic Xe applying a new technique of isotopically resolved photoelectron imaging. Selecting isotopes with zero nuclear spin, we were able to eliminate coupling of the electron angular momentum with the nuclear spin and highlight the pure electron dynamics. The PADs were measured across the $4 f^{\prime}$ autoionization resonance and the corresponding depolarization-free data for the asymmetry parameters provide a sound experimental basis for comparison with theory. The latter is in good agreement with the data, revealing high sensitivity of the asymmetry parameters to the details of the atomic structure and dynamics of the resonant photoionization.

We are grateful to the general SOLEIL staff for smoothly running the facility, and for beam provision under Project No. 20100271. The work was partially supported by the European COST Action CM1204 XLIC, the Russian Foundation for Basic Research (Grant No. 1202-01123), and the German Academic Exchange Service (joint DAAD and MSU program "Vladimir Vernadsky"). E. V. G. gratefully acknowledges support by the Russian President Grant No. MK-6509.2012.2 and by the Dynasty Foundation via the Support Program for Young Scientists. The authors also thank Catherine Le Bris for technical assistance in setting up the laser.

[1] U. Fano, Phys. Rev. 124, 1866 (1961).

[2] H. J. Wörner, M. Grütter, E. Vliegen, and F. Merkt, Phys. Rev. A 71, 052504 (2005).

[3] M. Schäfer, M. Raunhardt, and F. Merkt, Phys. Rev. A 81, 032514 (2010).

[4] F. Wuilleumier and M. Meyer, J. Phys. B 39, R425 (2006).

[5] B. Doughty, L. H. Haber, C. Hackett, and S. R. Leone, J. Chem. Phys. 134, 094307 (2011).

[6] V. Sukhorukov, I. Petrov, M. Schäfer, F. Merkt, M.-W. Ruf, and H. Hotop, J. Phys. B 45, 092001 (2012).

[7] U. Heinzmann and N. A. Cherepkov, VUV and Soft X-Ray Photoionization Studies, edited by U. Becker and D. A. Shirley (Plenum, New York, 1997), Chap. 15, pp. 521.

[8] K. L. Reid, Annu. Rev. Phys. Chem. 54, 397 (2003).

[9] C. Ott, A. Kaldun, P. Raith, K. Meyer, M. Laux, J. Evers, C. H. Keitel, C. H. Greene, and T. Pfeifer, Science 340, 716 (2013).

[10] M. P. Strand, J. Hansen, R.-L. Chien, and R. Berry, Chem. Phys. Lett. 59, 205 (1978).

[11] R.-l. Chien, O. C. Mullins, and R. S. Berry, Phys. Rev. A 28, 2078 (1983).

[12] L.E. Cuéllar, C. S. Feigerle, H. S. Carman, and R. N. Compton, Phys. Rev. A 43, 6437 (1991).

[13] K. L. Reid, S. P. Duxon, and M. Towrie, Chem. Phys. Lett. 228, 351 (1994).

[14] C. S. Feigerle, R. N. Compton, L. E. Cuéllar, N. A. Cherepkov, and L. V. Chernysheva, Phys. Rev. A 53, 4183 (1996).

[15] K. W. McLaughlin, K. Aflatooni, and D. W. Duquette, Phys. Rev. A 55, 3615 (1997). 
[16] R. P. Wood, C. H. Greene, and D. Armstrong, Phys. Rev. A 47, 229 (1993).

[17] J. R. Tolsma, D. J. Haxton, C. H. Greene, R. Yamazaki, and D. S. Elliott, Phys. Rev. A 80, 033401 (2009).

[18] E. Träbert, P. Beiersdorfer, and G. V. Brown, Phys. Rev. Lett. 98, 263001 (2007).

[19] In the $j K$-coupling scheme, the $n l[K]_{J}$ indicates, for the $\mathrm{Xe}$ atom, that the total angular momentum $\mathbf{j}$ of the $5 p_{j}^{5}$ core is first coupled to the orbital momentum of the excited electron $\mathbf{l}, \mathbf{j}+\mathbf{l}=\mathbf{K}$, with subsequent coupling of spin of this electron, $\mathbf{K}+\mathbf{s}=\mathbf{J}$. Primed and not primed orbitals of the excited electron correspond to $j=\frac{1}{2}$ and $j=\frac{3}{2}$, respectively.

[20] S. Aloïse, P. O'Keeffe, D. Cubaynes, M. Meyer, and A. N. Grum-Grzhimailo, Phys. Rev. Lett. 94, 223002 (2005).

[21] M. Meyer, M. Gisselbrecht, A. Marquette, C. Delisle, M. Larzillire, I. D. Petrov, N. V. Demekhina, and V.L. Sukhorukov, J. Phys. B 38, 285 (2005).

[22] L. Nahon, N. de Oliveira, G. A. Garcia, J.-F. Gil, B. Pilette, O. Marcouillé, B. Lagarde, and F. Polack, J. Synchrotron Radiat. 19, 508 (2012).
[23] G. A. Garcia, H. Soldi-Lose, and L. Nahon, Rev. Sci. Instrum. 80, 023102 (2009).

[24] G. A. Garcia, L. Nahon, and I. Powis, Rev. Sci. Instrum. 75, 4989 (2004).

[25] S. Baier, A. N. Grum-Grzhimailo, and N. M. Kabachnik, J. Phys. B 27, 3363 (1994).

[26] For a state with angular momentum $J=1$, the orientation and alignment parameters are defined as $\mathcal{A}_{10}=$ $\sqrt{\frac{3}{2}}\left(N_{+1}-N_{-1}\right)$ and $\mathcal{A}_{20}=\left(N_{+1}+N_{-1}-2 N_{0}\right) / \sqrt{2}$, respectively, where $N_{m}$ is the relative population of magnetic substate with the projection $m ; \sum_{m} N_{m}=1$. In the absence of the depolarization and assuming excitation of the electronic shell by a single ideally polarized photon, the alignment and orientation parameters take the strict values $\mathcal{A}_{10}^{+}=\sqrt{\frac{3}{2}}, \mathcal{A}_{20}^{+}=\frac{1}{\sqrt{2}}, \mathcal{A}_{10}^{\text {lin }}=0, \mathcal{A}_{20}^{\text {lin }}=-\sqrt{2}$.

[27] C. F. Fischer, T. Brage, and P. Jönsson, Computational Atomic Structure: An MCHF Approach (Institute of Physics Publishing, Bristol, 1997).

[28] A. N. Grum-Grzhimailo, S. Fritzsche, P. O'Keeffe, and M. Meyer, J. Phys. B 38, 2545 (2005). 